\title{
PHÄNOMENOLOGIE DES LEIBES BEI EDITH STEIN UND HERMANN SCHMITZ
}

Im Zentrum dieses Beitrages sollte eigentlich die Frage des $\mathrm{Zu}-$ gangs zum Mitmenschen stehen. Bei der Ausarbeitung bemerkte ich jedoch, dass für die Beantwortung dieser Frage die unterschiedlichen Konzeptionen des Leibes und die sich daraus ergebenden Konsequenzen von entscheidender Bedeutung sind, weshalb ich mich im folgenden auf diese Konzeptionen im Hinblick auf ihre Unterschiede und Gemeinsamkeiten konzentrieren möchte. Als Ausgangspunkt für meine Überlegungen dienen mir hauptsächlich Edith Steins „Zum Problem der Einfühlung” und Hermann Schmitz” "System der Philosophie".

Zunächst kann man konstatieren, dass für Edith Stein die Begriffe „Seele”, ,Leib” und „Körper” für die Bestimmung menschlicher Selbsterfahrung und Subjektivität von zentraler Bedeutung sind ${ }^{1}$, während Hermann Schmitz den Begriff der "Seele" aufgibt, weil er der Überzeugung ist, man könne phänomenologisch nicht sinnvoll von ihr sprechen².

${ }^{1}$ Vgl. vor allem E. Stein, Zum Problem der Einfühlung, Halle 1917, S. 43-101. Dass auch Begriffe wie „Bewusstseinsstrom”, „Ich”, „Subjektivität”, „Person” und "Geist” von zentraler Bedeutung sind, möchte ich damit natürlich nicht sagen. Mir geht es hier nur um eine Auswahl von Beschreibungsperspektiven, mit der man - meiner Meinung nach - wichtige Unterschiede zu fassen bekommt und mit deren Hilfe man Differenzen gut herausarbeiten kann.

2 Obwohl H. Schmitz in den ersten zwei Bänden seines "Systems der Philosophie" noch von der "Seele" spricht. Ab Band III spielt sie jedoch keine Rolle mehr, später nimmt er explizit Distanz dazu (vgl. etwa H. Schmitz, Der Leib, Berlin 2011, S. 5: „Nach meiner Überzeugung ist 
Für Edith Stein ist die Seele eine „substanzielle Einheit”, die unseren Erlebnissen als „Träger” zugrunde liegt und sich z.B. durch die Schärfe unserer Sinne, die Energie unseres Handelns bekundet ${ }^{3}$. Diese Seele jedoch „ist notwendig immer Seele in einem Leib" 4 . Wie sie sich von diesem unterscheidet und worin dessen Differenz zum Körper besteht, ist ein phänomenologisches Kernstück der Theorie Edith Steins und soll im folgenden Thema sein.

Für sie tritt der Unterschied zwischen Körper und Leib am deutlichsten hervor, wenn man versucht, sich selbst mit den eigenen Sinnen wahrzunehmen. Während ich jedes andere Objekt mehr oder weniger vollständig aus verschiedenen Perspektiven wahrnehmen kann, steht es beim eigenen Körper anders, denn hier „wälzen” die Sinne, wie Edith Stein es für Tastund Gesichtssinn formuliert, "gegenseitig die Verantwortung aufeinander $\mathrm{ab}^{\prime \prime}$. Der eigene Körper ist mir also auf eine andere Art und Weise gegeben als alle anderen Objekte. Dass Edith Stein hier auf mehr hinauswill als nur auf den eigenen Körper in der Wahrnehmung, wird daran ersichtlich, dass sie betont, dass es sich hierbei um ein Objekt handelt, zu dem ich mich nicht positionieren kann: „Es ist immer «hier», während alle anderen Objekte immer «dort» sind"6. Das gilt sowohl' für den eigenen Körper, von dem ich nicht abrücken kann, viel mehr aber noch für das, was sie „Leib” nennt. Dieser ist das, was ich selbst dann spüre, wenn ich die Augen schließe und meine Tastempfindungen auf ein Minimum reduziere.

Obwohl es Edith Stein meiner Meinung nach nur schwer gelingt, hier eine begrifflich und phänomenologisch scharfe Trennung vorzunehmen, entdeckt sie etwas, das von großer Bedeutung ist: „«Nullpunkt der Orientierung», den mein Leib umgibt" ${ }^{\text {" }}$. Dieser sei für alle Wahrnehmungsdaten ein anderer - für die visuellen im Kopf, für die taktilen im Zentralleib; das ,Ich' habe außerdem keine Distanz zu diesem Nullpunkt. Zum anderen beschreibt sie die "Einheit des Leibes”, die sie aus der Beobachtung der

\footnotetext{
die Seele nichts Vorfindbares, sondern ein ideologisches Konstrukt, von den Griechen ausgedacht, um das gesamte Erleben eines Menschen in einer privaten Innenwelt unterzubringen, damit er als Vernunft Herr im eigenen Hause über die unwillkürlichen Regungen, namentlich die leiblichen und die durch sie eingreifenden Gefühle, werden kann”; oder H. Schmitz, „System der Philosophie V, Die Aufhebung der Gegenwart“ Bonn 1980, S. 191: „Was den Tod überdauert, kann nicht die Seele sein, denn die gibt es nicht, wohl aber der spürbare Leib, der weder sicht- und tastbarer Körper ist, noch ausdehnungs- und ortlose Seele".

${ }^{3}$ Vgl. Edith Stein, Zum Problem der Einfühlung, S. 43.

4 Ebenda, S. 44.

${ }^{5}$ Vgl. ebenda, S. 45.

${ }^{6}$ Ebenda.

${ }^{7}$ Edith Stein, Zum Problem der Einfühlung, S. 46.
} 
Empfindungen abliest. Alle diese Empfindungen haben gemeinsam, dass sie auf die Frage „wo?" antworten und nahe beim „Ich" gelegen seien, das eigentlich „unräumlich" und nicht zu lokalisieren" sei ${ }^{8}$. Sie spricht also einerseits vom "Leib als Ganzem", betont andererseits jedoch auch die Differenzierungen, die bezüglich dieses Ganzen festzustellen sind.

Damit scheint sie mir in ein Spannungsfeld zu geraten, aus dem erst mit der Phänomenologie Hermann Schmitz' ein Ausweg gefunden ist. Doch dazu später. Festzuhalten ist zunächst das Positive und dazu gehört neben dem bereits Gesagten eben auch die Feststellung, dass es so etwas wie einen "Leibraum" und einen "Außenraum" gibt, die mir auf fundamental unterschiedliche Weise gegeben sind, sich aber unter Umständen überschneiden können ${ }^{9}$. Beispielhaft dafür sind die unterschiedlichen Zugangsweisen zum eigenen Leib, der mir als „empfindender (leibwahrgenommener) Leib und äußerlich wahrgenommener Körper der Außenwelt"10 gegeben ist.

Der sogenannte Leibraum wird besonders dann auffällig, wenn ich mich im Außenraum bewege, denn dann nehme ich das „Hier” meiner Empfindungen und auch mein "Ich” quasi mit mir. Ich werde mein „Hier” also niemals los. Diese Beobachtung scheint mir auf das zu verweisen, was Hermann Schmitz später den "absoluten Ort” nennen wird. Doch während Edith Stein davon ausgeht, dass "die Möglichkeit eines Ich ohne Leib"11 besteht, bindet Hermann Schmitz die Subjektivität genau an den Leibbegriff und vermeidet die Rede vom „Ich”. Bevor ich mich jedoch Schmitz' Phänomenologie als Vergleichspunkt zuwende, möchte ich noch auf einen letzten Aspekt in Edith Steins Theorie eingehen: den Zusammenhang des Leibes mit den Gefühlen.

Die sinnlichen Gefühle oder Gefühlsempfindungen verortet sie zum einen dort, wo die Empfindung verspürt wird, zum anderen geht sie davon aus, daß diese dem Ich „entquellen”12. Ähnlich fällt ihre Beschreibung der sogenannten "Gemeingefühle" - z. B. Mattigkeit oder Frische - aus, die nicht nur „das Ich” füllten, sondern „in allen Gliedern” gespürt würden. Interessanterweise beharrt sie auch hier auf eine Trennung von "Ich" und „Leib”. Die Gemeingefühle würden außerdem immer als vom Leib herkommend erlebt werden und das Erleben des Menschen als Ganzen affizieren. Diese Beschreibung kennen wir auch aus anderen - zum Teil früheren, zum Teil späteren - Theorien, wichtig ist mir in diesem Zusammenhang, dass Edith

\footnotetext{
8 Ebenda.

${ }^{9}$ Vgl. ebenda.

${ }^{10}$ Ebenda.

11 Ebenda, S. 52.

12 E. Stein, Zum Problem der Einfühlung, S. 53.
} 
Stein auch nichtleibliche Gemeingefühle (die "Stimmungen”, wie z. B. Heiterkeit oder Trübsinn) benennt und diese der Seele zuordnet. So kommt sie zu dem Schluss, dass es seelische und leibliche Gemeingefühle gebe, die sich gegenseitig beeinflussen, befördern oder behindern können.

Dies soll mir als kurzer Aufriss genügen, den ich nun der Phänomenologie Hermann Schmitz' gegenüberstellen möchte. Hierbei werden frappierende Ähnlichkeiten sichtbar werden, aber auch ebenso deutliche Unterschiede, die gerade wegen der Verwendung desselben Vokabulars vielleicht auch überraschen mögen.

Schmitz trennt zunächst „Körper” und „Leib” begrifflich so voneinander, daß er den Leib als das bestimmt, was man von sich ohne ein "Sinnesorgan" spüren kann ${ }^{13}$. Wenn man also - ganz analog zu dem Vorschlag Edith Steins - versucht, sich zu spüren, „entdeckt” man nach Schmitz' Auffassung den Leib. Schmitz geht nun aber noch einen Schritt weiter. Er nimmt diesen Spürversuch ernst und findet so die verschiedene Strukturierung von Körper und Leib. Während der Körper durch nahtlose Kontinuität gekennzeichnet ist, bietet sich der Leib dem Spürenden zwar ebenfalls ein Ganzer dar, aber nicht kontinuierlich, sondern als „Gewoge verschwommener Leibesinseln"14. Unser perzeptives Körperschema deckt sich also offensichtlich nicht mit unserem (eigen-)leiblichen Empfinden. Schmitz gelingt es hier anders als Edith Stein, die allerdings schon in diese Richtung weist - eine scharfe Differenzierung von Körper und Leib vorzunehmen. Der Körper ist

${ }^{13}$ H. Schmitz, Der unerschöpfliche Gegenstand, Bonn 1990, S. 115: „Wenn ich vom Leib spreche, denke ich nicht an den menschlichen oder tierischen Körper, den man besichtigen und betasten kann, sondern an das, was man in dessen Gegend von sich spürt, ohne über ein «Sinnesorgan» wie Augen oder Hand zu verfügen, das man zum Zweck dieses Spürens willkürlich einsetzen könnte. Das so Gespürte bezeichne ich als leibliche Regung; es kann die Grenzen des sicht- und tastbaren Körpers überschreiten, wie im Fall der Phantomglieder der Amputierten. Schmerz, Hunger, Durst, Schreck, Wollust, Frische, Mattigkeit, Behagen, Ein- und Ausatmen sind Beispiele leiblicher Regungen, die am eigenen Leib gespürt werden; ich spreche in diesem Sinn vom eigenleiblichen Spüren und dessen Gegenstandsgebiet".

${ }^{14}$ H. Schmitz, Leib und Gefühl. Materialien zu einer philosophischen Therapeutik, Paderborn 1989, S. 42: "Statt stetigen Zusammenhangs begegnet dem Spürenden ein Gewoge verschwommener Inseln in größerer oder geringerer Zahl, dünnerer oder dichterer Verteilung. Sie befinden sich in beständiger, gewöhnlich fast unbemerkter Wandlung, ermangeln des scharfen Umrisses und der beharrlichen Lagerung. Man kann das Experiment auch an einzelnen Gliedern ausführen, z.B. am Fuß. Optische und taktile Wahrnehmung liefern die bekannte Gliederung zwischen Hacke und Zehen. Für das eigenleibliche Spüren pflegt dagegen die gestalthafte Einheit des Fußes zu fehlen. Die Gegenden des Fußgelenks, der Fußknöchel und der Sohlen bilden eigene Leibesinseln und verschmelzen zu einem Gesamtfuß höchstens, wenn - etwa nach übermäßiger Gehanstrengung - «der Fuß geschwollen` ist und als diffuse, müde, dumpfe, taube, schmerzhafte Masse gespürt wird". Vgl. H. Schmitz, System der Philosophie II/1: Der Leib, Bonn 1965, S. 12-35. 
durch relative Örtlichkeit bestimmt, während der Leib absolut örtlich ist ${ }^{15}$. Die Einheit des Leibes als absoluter Ort trotz dem Vorhandensein verschiedener Leibesinseln ist also dadurch gewährt, dass die Leibesinseln diesen absoluten Ort zugleich darstellen und ihn sich teilen ${ }^{16}$.

Die Lösung für ein Problem, vor dem Edith Stein noch suchend steht, scheint Schmitz in der Unterscheidung eines absolut-örtlich bestimmten ,reinen Leibes', der aber nur unter besonderen Umständen spürbar ist und eines ",körperlichen Leibes”, der auch relativ-örtlich bestimmt ist, gefunden zu haben. Der "körperliche Leib" ist Leib in der Hinsicht, dass er nicht zerlegbar ist; während er Körper in der Hinsicht ist, dass er einen Ort in einem Gefüge von Lage- und Abstandsbeziehungen einnimmt, der sich nicht in einem „Hier" erschöpft, sondern ein „Hier" mit Bezug zu anderen Punkten bzw. Orten in einem Raum ist. Die Parallelen zu Edith Stein sind unübersehbar, die von einem „Nullpunkt der Orientierung" spricht, der ein „Hier" darstellt, das wir sozusagen mit uns herumtragen.

Schmitz' Zuschreibung der verschiedenen Weise der Örtlichkeit beruht auf der von ihm entwickelten und zugrunde gelegten Raumtheorie, in der er drei Weisen des leiblichen Raumes voneinander unterscheidet: den Weiteraum, den Richtungsraum und schließlich den Ortsraum ${ }^{17}$. Auf die Details kann ich hier aus Platzgründen leider nicht eingehen ${ }^{18}$.

${ }^{15}$ H. Schmitz, System der Philosophie II/1: Der Leib, S. 54: „Der reine Leib ist bloß absolutörtlich und gar nicht relativ-örtlich bestimmt; er kommt bei den panischen Zuständen von Angst, Schmerz und Wollust vor, wenn die räumliche Orientierung verloren gegangen ist. Der reine Körper ist bloß relativ-örtlich und gar nicht absolut-örtlich bestimmt; er bildet das Objekt der naturwissenschaftlichen Beschäftigung von Anatomie, Physiologie und exakt messender Medizin mit dem menschlichen Körper. In der Mitte zwischen beiden steht der körperliche Leib, der sowohl absolut-örtlich als auch relativ-örtlich ist: das Gewoge verschwommener Inseln, die ebenso je für sich einen relativen und einen absoluten Ort haben, wie sie durch einen absoluten Ort zur Einheit des Leibes im Ganzen zusammengehalten werden. [...] Um die Frage zu entscheiden, ob ein gegebenes Phänomen dem körperliche Leib oder dem reinen Körper angehört, ist [...] nur zu prüfen, ob es unteilbar oder teilbar ausgedehnt ist".

16 Ebenda, S. 46: „[...] dass dieser absolute Ort ganz im ganzen körperlichen Leib und auch ganz auf jeder Leibesinsel vorhanden ist. [...] Mit der [...] Behauptung, dass jener absolute Ort auch auf jeder Leibesinsel ganz vorhanden ist - ist gemeint: Er ist auf jeder Leibesinsel vorhanden, da er alle diese Inseln umfasst; auf keiner von ihnen ist aber nur ein Teil von ihm vorhanden, da er nicht teilbar ist”. Vgl. aber auch S. 38: „[...] so weit, wie sich eigene Leibesinseln erstrecken, auch der absolute Ort des eigenen Leibes im Ganzen reicht”. S. 39: „Der neue Beweis aus der Einheit des Leibes liefert darüber hinaus die Einsicht, dass alle diese absoluten Orte von Leibesinseln überformt und zusammengefasst sind durch einen absoluten Ort des Leibes im Ganzen".

17 Vgl. dazu vor allem H. Schmitz, System der Philosophie III/1: Der Raum. Der leibliche Raum, Bonn 1998 (1967), S. 7-10, S. 28-130 u. ö.; und ders., System der Philosophie III/2: Der Raum. Der Gefühlsraum, Bonn 1969, S. 185-360, u. ö.

${ }_{18}$ Die Frage, was Raum eigentlich sei, beantwortet er so: „Raum ist Weite in reiner oder überformter Gestalt”. Das Gegenteil dieser Weite ist die Enge, die Schmitz als „den Zusam- 
Der absolute Ort, durch den der reine Leib oben bestimmt worden war, ist nicht nur der Ausgangspunkt jeder räumlichen Bestimmung oder Orientierung19, sondern zugleich auch die Quelle des Subjekts ${ }^{20}$. „Leib” und „Subjektivität" stehen sich also nicht gegenüber, wie "Leib" und „Ich" in Edith Steins Theorie, sondern fallen gewissermaßen ineinander. In Schmitz Worten ist das „Hier" des Leibes:

für jeden jeweils unverwechselbar als der absolute Ort bestimmt, wo er sich selbst spürt, während alle anderen Orte einem vieldeutigen Anderswo und Irgendwo angehören. Der absolute Ort, wo jemand sich leiblich findet, ist für ihn primär nie „irgendwo", d. h. vieldeutig und genauerer Besinnung bedürftig, sondern er ist die ohne Rücksicht auf Lagen und Abstände zu anderen Orten eindeutig bestimmte Quelle aller Lokalisierung, die bei den Umgebungsdingen erst durch deren Bindung an den eigenen ausgezeichneten Ort möglich wird ${ }^{21}$.

Während sich bei Edith Stein „Leib” und „Körper” auf das gleiche Objekt beziehen, sind „Leib” und „Körper” nach Schmitz streng zu unterscheiden, auch wenn sie unter Umständen den gleichen Raum einnehmen können.

Worin jedoch die Verbindung von Leib und Körper besteht, ist ein Grundproblem, das Schmitz letztlich nicht aufzulösen vermag. Obwohl er immer wieder die Verbindung von Leib und Körper aufzeigt, bleibt es für ihn ein Rätsel, wie diese phänomenologisch zu erklären ist.

Ein weiterer Vergleichspunkt zu Edith Stein ist Schmitz Thematisierung der Gefühle. Schmitz bestimmt diese als „ortlos räumlich ergossene Atmosphären" 22 und greift bei dieser Bestimmung auf seine Raumtheorie zurück. Seine Gefühlstheorie will die 〈Innenwelt〉 verschwinden lassen und lässt den Menschen durch dessen „In-der-Welt-Sein” 23 zu einer Art Resonanzboden für die ihn umgebenden "Atmosphären" werden. Gefühle sind somit, wie Schmitz es formuliert, ",nicht subjektiver als Landstraßen” ${ }^{24}$. Erst durch un-

menfall der fünf Momente Hier, Jetzt, Dasein, Dieses und Ich auch als «primitive Gegenwart» bezeichnet". H. Schmitz, System der Philosophie III/1: Der Raum. Der leibliche Raum, S. 12.

${ }^{19}$ Ebenda, S. 13.

20 Vgl. H. Schmitz, System der Philosophie IV: Die Person, Bonn 1990 (1980), S. 12-27 und passim.

${ }^{21}$ H. Schmitz, System der Philosophie III/1: Der Raum. Der leibliche Raum, S. 13; Philosophie III/2: Der Raum. Der Gefühlsraum, S. 87f

22 Vgl. Ebenda, S. 343.

${ }^{23}$ Ein Ansatz, der spätestens seit Martin Heideggers „Sein und Zeit” wirkmächtig geworden ist. Vgl. z. B. M. Heidegger, Sein und Zeit, Tübingen 1993 (1927), S. 52f.

${ }^{24}$ H. Schmitz, System der Philosophie I: Die Gegenwart, Bonn 1998 (1964), S. 144; und ders., System der Philosophie III/2: Der Raum. Der Gefühlsraum, S. 87f. 
ser affektives Betroffensein von ihnen werden sie zu unseren Gefühlen. Das affektive Betroffensein durch Gefühle unterscheidet sich zwar von leiblichen Regungen, ist jedoch an diese gebunden und von diesen vermittelt ${ }^{25}$. Als leibliche Wesen werden wir von den uns umgebenden Atmosphären gelenkt, sind diesen jedoch nicht willenlos ausgeliefert; es ist sogar möglich, ein Gefühl wahrzunehmen, ohne es zu fühlen. Erst wenn wir von einem Gefühl ergriffen werden, fühlen wir es auch ${ }^{26}$.

Durch die Gefühle sind wir also beständig dazu aufgefordert, uns in irgendeiner Weise zu ihnen zu verhalten, wobei wir Impulse und Lenkung durch sie erfahren. Je mehr wir die ganzheitlich wahrgenommenen Situationen durch Explikation analysieren und vereinzeln, desto weniger sind wir dem Ergriffensein durch sie ausgeliefert. Hierin besteht zugleich ein Gewinn und ein Verlust, wie Schmitz konstatiert, denn mit der Überführung der Situationen in handhabbare Konstellationen geht die Gefahr einher, die Rolle des Menschen zu überschätzen und die Bedeutung der Situationen und leiblichen Regungen für unsere innerweltliche Orientierung zu übersehen ${ }^{27}$. Im Gegensatz zu Edith Stein „entquellen” die Gefühle in Schmitz' Theorie also nicht dem Leib oder einem Ich, sondern sind überpersönlich. Ganz ana$\log \mathrm{zu}$ Stein beschreibt Schmitz allerdings die eigenleiblichen Regungen wie Mattigkeit oder Frische, die bei ihr als „Gemeingefühle” verhandelt werden.

Auch bezüglich der Wahrnehmung lässt sich feststellen, dass Schmitz ausgehend von seiner Theorie des Leibes die Ketten der Tradition abschüttelt und so lautet seine Definition der Wahrnehmung simpel: „Wahrnehmung ist [...] Gegebenheit oder Sichpräsentieren von etwas in leiblicher Kommunikation" 28 .

Von leiblicher Kommunikation spricht Schmitz, „wenn jemand von etwas in einer für ihn leiblichen Weise so betroffen und heimgesucht wird, dass er mehr oder weniger in dessen Bann gerät und mindestens in Versu-

${ }^{25}$ Vgl. Ebenda, S. 153f.

${ }^{26}$ Ebenda, S. 144: „Das affektive Betroffensein von Gefühlen hat also einen spannungsreichen, dialogisch-dynamischen Charakter, und das Gefühl ist dabei nicht Zustand, sondern Gegenspieler des Betroffenen. Die Auseinandersetzung beider Parteien braucht nicht auffällig und heftig zu sein, sondern reicht von wilden Konflikten bis zu den sanftesten Schwingungen; immer bietet sich dabei dem betroffenen Subjekt nach der anfänglichen Überwältigung ein Spielraum möglicher Stellungnahmen in der Dimension von Hingabe und Abwehr, Öffnung und Verschluss an".

${ }^{27}$ Für die mit der Autorität der Gefühle zusammenhängenden ethischen Problemfelder vgl. etwa H. Schmitz, Der unerschöpfliche Gegenstand, S. 334-362.

28 Ders., System der Philosophie III/5: Der Raum. Die Wahrnehmung, Bonn 1989 (1978). Zu einer präzisen Bestimmung dessen, was leibliche Kommunikation ist siehe z. B. ebd., S. 31f. oder ders., Der unerschöpfliche Gegenstand, S. 135-140. 
chung ist, sich danach zu richten" ${ }^{29}$. Zum einen ist Wahrnehmung in Schmitz' Verständnis also weniger als ein aktiver Prozess seitens des Wahrnehmenden gedacht, sondern dieser wird von dem, was er wahrnimmt, eher gelenkt ${ }^{30}$. Das heißt nicht, dass Wahrnehmung in diesem Sinn eine bloße Aufnahme oder Verarbeitung von Signalen wäre ${ }^{31}$. Im Gegenteil ist Wahrnehmung als eine Art Koagieren von Wahrnehmendem und Wahrgenommenem zu verstehen.

Weiterhin fällt ins Auge, dass in Schmitz' Definition der Wahrnehmung keine Rede von Sinnesorganen ist. Diese betrachtet er nicht als Vermittler, Werkzeuge oder Schleusen der Wahrnehmung - wie Edith Stein dies zu tun scheint - sondern bloß als Stätten einer sich mit der Wahrnehmung "regelmäßig verbindenden Begleitmusik”, "die sich in keinem ernstlich und nüchtern belegbaren Sinn je «zwischen» das Wahrnehmen und das Wahrgenommene zu schieben vermag" 32 . Zwar geht er davon aus, dass die Sinnesorgane eine notwendige Bedingung des Wahrnehmens sind, doch folgt daraus für ihn eben nicht, dass wir mit den Augen sehen bzw. mit den Ohren hören ${ }^{33}$. Damit richtet er sich gegen einen Physiologismus und Sensualismus, der durch die Annahme von Sinneskanälen verkennt, dass im Wahrnehmen ganzheitliche Situationen erfasst werden ${ }^{34}$, die erst nachträglich in Einzelinformationen zergliedert werden. Die Wahrnehmung ist also primär leiblich, in dem Sinn, dass der Blick als leibliche Regung verstanden keine Entsprechung im Körper hat. Die Augen kommen beim Erfahren des Sehens nicht vor, wohl aber der Blick, sowohl der eigene, den man schweifen oder auf etwas ruhen lassen kann, als auch der fremde, der einen trifft.

Abschließend sei mir noch eine Beobachtung zum von Edith Stein beschriebenen Verhältnis von "Ich" und „Leib” gestattet, von dem sie ja sagte, dass die Möglichkeit eines Ichs ohne Leib bestünde. Für Schmitz stellt sich dieser Sachverhalt genau umgekehrt dar. Ausgehend von Beschreibungen des Phänomens der Phantomgliederlebnisse oder sogenannter Heautoskopien nimmt er an, dass abgespaltene Leibesinseln bzw. der „Leib

${ }^{29}$ H. Schmitz, System der Philosophie III/5: Der Raum. Die Wahrnehmung, S. 31f.

${ }^{30}$ Vgl. ders., Neue Grundlagen der Erkenntnistheorie, Bonn 1994, S. $126 \mathrm{f}$.

${ }^{31}$ Vgl. dazu auch ders., Der unerschöpfliche Gegenstand, S. 138f.

32 Ders., System der Philosophie III/5: Der Raum. Die Wahrnehmung, S. 4.

${ }^{33}$ Vgl. dazu auch ders., Neue Grundlagen der Erkenntnistheorie, S. 2 f.

${ }^{34}$ Zum Situationsbegriff vgl. etwa Hermann Schmitz, System der Philosophie IV: Die Person, S. 14f: „Eine Situation überhaupt ist eine absolut oder relativ chaotisch-mannigfaltige Ganzheit, zu der mindestens Sachverhalte gehören". Außerdem ders., System der Philosophie III/4: Der Raum. Das Göttliche und der Raum, Bonn 1995 (1977), S. 411-444; und ders., Situationen und Konstellationen. Wider die Ideologie der totalen Vernetzung, Freiburg 2004. 
im Ganzen" 35 ohne den reinen Körper vorkommen oder diesen sogar wechseln können ${ }^{36}$. Schmitz hält daher die Annahme einer „Unsterblichkeit des Leibes bei Trennung vom reinen Körper, aber mit räumlicher, örtlich umschriebener Ausdehnung und bei [...] Bewusstsein"37 nicht nur für möglich, sondern für vernünftig.

Dies kann nur eine vorläufige Skizze sein. Ich hoffe aber gezeigt zu haben, dass ein etwas genauerer Blick auf die den Theorien zugrunde liegenden Phänomene sowohl Gemeinsamkeiten als auch Unterschiede dort offenbart, wo man sie vielleicht nicht erwarten würde.

\section{Phenomenology of the Body (Leib) according to Edith Stein and Hermann Schmitz}

Summary

Steffen Kammler carries out a detailed analysis of phenomenology of the body contained in Edith Stein's dissertation On the Problem of Empathy and Hermann Schmitz's Philosphical System (System der Philosophie), highlighting similarities and deep differences in their views. The author shows the relevance of Edith Stein's philosophical approach to these issues in dialogue with the newest trends in phenomenological studies and reveals the stimulating influence of Stein's way of thinking about body (Leib) on German phenomenology in the second half of the twentieth century.

35 Diese Formulierung spricht meines Erachtens gegen Soentgens Auffassung, dass man den Leib (den es strenggenommen nicht gebe, sondern nur leibliches Befinden) in keiner Weise als Substanz verstehen dürfe. Vgl. J. Soentgen, Die verdeckte Wirklichkeit, Bonn 1998, S. 61f.) Schmitz selbst jedenfalls scheint dies zu tun.

36 Vgl. ders., System der Philosophie V: Die Aufhebung der Gegenwart, S. 191. Schmitz spricht dort sogar von der Möglichkeit von Gespenstern, die als Leiber ohne reinen Körper existieren könnten und nicht seh-, hör- bzw. tastbar sind.

${ }^{37}$ H. Schmitz, System der Philosophie V: Die Aufhebung der Gegenwart, S. 192. 